\section{Solar oblateness}

from David W. Hughes

THE Sun is thought to be flattened. Dicke and Goldenberg (Astrophys. J. Suppl., 27, 131; 1974) gave (4.51士 $0.34) \times 10^{-5}$ as the value for this oblateness which means that the polar radius is $31.4 \mathrm{~km}$ smaller than the equatorial radius. Not much considering the mean solar radius is $695,997 \mathrm{~km}$. Now it is clear that a rapidly rotating solar core with a period of between 0.5 and $2 \mathrm{~d}$ could provide an explanation for this flattening; but what is not clear is why the core should rotate so rapidly. This problem has been approached theoretically in a recent article by Schatten (Astrophys. Space Sci., 34, 467; 1975).

Obviously there must be some interaction between the quickly rotating core and the slowly rotating photosphere. Because the solar interior contains essentially all the mass and the angular momentum the photosphere would be spun up to the angular velocity of the interior unless there were some braking force acting on it. Dicke suggested that it is the accelerating torque exerted on the solar wind by the magnetic fields locked in the photosphere which provide this brake. In his first approach to the problem Schatten assumes that the rate of change of angular velocity with time is negligible and then finds that the angular velocity of the solar core is simply obtained by dividing the solar wind's torque, $\tau_{\mathrm{Sw}}$, by the rate of change of the solar moment of inertia with time $\mathrm{d} I / \mathrm{d} t$. This moment of inertia varies because the nuclear reaction in which hydrogen is converted into helium reduces the number of ionised particles in the solar interior. To conserve the number of particles per unit volume and the pressure balance the core has to contract. Taking $\tau_{\mathrm{sw}}$ as $-8 \times 10^{30}$ dyne $\mathrm{cm}$, calculations of $\mathrm{d} I / \mathrm{d} t$ as a function of $r$, the distance from the centre of the Sun, gives a rotation period of $17.5 \mathrm{~h}$ for $r<117,000 \mathrm{~km}$ and $25.4\left(r / R_{\odot}\right)^{2}$ days for $r>117,000 \mathrm{~km}$.

In Schatten's second approach the square of the angular velocity of the core is found to equal the mean power loss due to solar activity, $P_{s_{A}}$, divided by $0.5 \mathrm{~d} I / \mathrm{d} t$. As the core contracts the rotation power is liberated through the magnetic fields in sunspots. The energy lost in a solar cycle is calculated to be $10^{35} \mathrm{erg}$, equivalent to $2 \times 10^{27} \mathrm{erg}$ $\mathrm{s}^{-1}$. However some of the spot energy might not get away from the Sun and Schatten, considering flares, gets a more dependable value of $10^{26} \mathrm{erg} \mathrm{s}^{-1}$. These two values give a core rotation period of between 1 and $4 \mathrm{~d}$. Third, the core rotation period must equal $\mathrm{P}_{\mathrm{SA}} / \tau_{\mathrm{SW}}$ which gives periods of 0.5 to $5 \mathrm{~d}$. These theoretical values of core rotational velocity, based upon simple principles of conservation of angular momentum, energy and torque flow from the Sun, give a total oblateness of $3.4 \times 10^{-5}$ for the photosphere which is in close agreement with the observed value of $4.5 \times 10^{-5}$.

Three interesting problems still remain. How does the solar core maintain its rapid rotation, bearing in mind that it is a highly conducting plasma? Also what effect does this oblateness have on celestial mechanics? The value obtained would produce a $4.05 \mathrm{~s}$ of arc per century advance in the perihelion of Mercury. This opens up the question of the accuracy of the general relativistic tensor theory of gravitation in comparison, say, with the scalar tensor theory. Third, can the rapid rotation of the core produce a lower central temperature in the Sun and thus help explain why the neutrino flux is less than expected?

It must be mentioned, however, that not everyone agrees with the oblateness values obtained by Dicke and Goldenberg. Hill and Stebbins in a paper scheduled for the September edition of The Astrophysical Journal discuss observations they have made at the University of Arizona's observatory in the Santa Catalina Mountains. The value they obtained for the oblateness is $(8.6 \pm 5.9) \times 10^{-6}$ which indicates that the Sun's shape is indistinguishable from that of a sphere.

\section{Nitrogen in synthetic diamond}

\section{from John Walker}

Diamonds might be a girl's best friend, but in many respects they are very puzzling to scientists. One of the most intriguing problems concerns nitrogen, which is a very common impurity in diamond, and which has recently been studied in synthetic crystals by some Soviet scientists (Yu. A. Klyuev, V. I. Nepsha, and A. M. Naletov, Sov. Phys. Solid State, 16, 2118; 1975). Nitrogen occurs in aggregated form in most natural diamonds, but in a few rare crystals the nitrogen atoms are isolated, each one replacing a single carbon atom. These crystals can be detected because the nitrogen is paramagnetic -it can be observed in magnetic resonance experiments.

Surprisingly enough, synthetic diamonds usually contain isolated paramagnetic nitrogen rather than clusters. To investigate this difference, and to check the earlier magnetic resonance work on natural diamond, Klyuev et al. have grown synthetic diamonds doped with the rare nitrogen isotope ${ }^{15} \mathrm{~N}$. This isotope has a nuclear spin of one-half, which gives rise to a magnetic

\section{HLA histocompatibility system}

In the article "Histocompatibility testing international" which appeared in the News and Views Section last week, the designation of the HLA system was changed during editing to HL-A. The WHO nomenclature committee mentioned in the report did in fact recommend that the whole region should now be designated HLA.

resonance spectrum somewhat different from the ${ }^{14} \mathrm{~N}$ which predominates in natural diamond and has a spin of unity. The Russian workers were able to confirm the earlier work, allowing for the different nuclear spin.

Nitrogen in diamond can also be detected using infrared spectroscopy. The absorption spectra of the ${ }^{15} \mathrm{~N}$ diamonds were very similar to those of ${ }^{14} \mathrm{~N}$ crystals, except that a peak at $1,135 \mathrm{~cm}^{-1}$ in the latter had shifted to $1,120 \mathrm{~cm}^{-1}$. This was accounted for by the different masses of the two isotopes. A second peak, at $1,282 \mathrm{~cm}^{-1}$, which can be caused by nitrogen clusters, was relatively strong in the doped crystals, irrespective of the isotope used. Undoped diamonds were also found to contain paramagnetic nitrogen, presumably as a result of contamination from the atmosphere, but had a relatively weak $1,282 \mathrm{~cm}^{-1}$ peak. This suggests that nitrogen clusters are present as a minor species in doped synthetic crystals.

The most likely cause of this difference in nitrogen's behaviour in natural and synthetic diamonds is that different pressures and temperatures are used in the two crystallisation processes. Laboratory studies by the same authors (Yu. A. Klyuev, Yu. A. Dudenkov, and V. I. Nepsha, Geo. chem. Int., 7, 781; 1973) have shown that relatively high pressures and low temperatures during the synthesis process create cubic crystals which contain a lot of dispersed nitrogen, but few aggregates. Lower pressures and higher temperatures create octahedral crystals with a lot of nitrogen clusters, very similar to natural diamonds. What is not clear is whether the aggregates are formed directly during the crystallisation process, or whether they grow afterwards, in the high pressures and temperatures needed for synthesis. The authors favour the latter hypothesis, but further work is needed to decide the issue. The fact that synthetic diamond can now be doped with a specific isotope indicates how well the process can be controlled. This has implications not only for diamond physicists, but also in the realm of new applications. 